\section{Predictive value of Gram staining of low respiratory tract secretions paired to routine culture}

\section{Valor predictivo de la tinción de Gram en secreciones del tracto respiratorio bajo}

\section{To the Editor:}

Microbiological methods such as Gram staining and culture of low respiratory tract secretions are necessary to detect the real bacterial pathogens that cause lower respiratory tract infections (LRTI). In many cases, Gram stain analysis may be unreliable to determine the cause of LRTI, and bacterial cultures provide adequate information ${ }^{1}$. However, we herein report two cases of lower respiratory tract infections, diagnosed with the aid of microscopic examination of Gram staining, while routine cultures were negative in $48 \mathrm{~h}$.

Case 1. A 74-year-old woman was referred to the hospital with the chief complaints of cough, fever, expectoration and episodes of hemoptysis lasting one month. A protected specimen brush (PSB) and a brochoalveolar lavage (BAL) were successively obtained. The samples were processed with Gram staining and routine cultures, done within $0.5 \mathrm{~h}$ of obtainment. On Gram staining, the organisms appeared as Gram-positive thin branching, beaded, coccoid filaments. Modified ZiehlNielsen staining (with $1 \%$ sulphuric acid) showed many acid-fast thin branching beaded filamentous structures, consistent with the morphology of Nocardia species. Meanwhile, the results of culture on blood agar and chocolate agar were negative at $37^{\circ} \mathrm{C}$ after $48 \mathrm{~h}$. Small, dry, wrinkled, irregular, yellowish white colonies appeared on blood agar and chocolate agar after $72 \mathrm{~h}$ of incubation, which was also identified as Nocardia asteroides by standard bacteriological methods ${ }^{2}$.

Case 2. A 42-year-old woman was diagnosed with systemic lupus erythematosus (SLE) in 2001 at the age of 32 years. In 2011, she was admitted to hospital with a two-week history of productive cough, wheezing, and fever. Culture and Gram staining of PSB and BLA were performed. Gram staining of all samples revealed a septate, hyphal organism, with dichotomous branching at an acute angle. Routine cultures at $48 \mathrm{~h}$ did not reveal any significant pathogens in the samples. At 72 hours of incubation, mycelial fronts of colonies grew on blood agar and chocolate agar. The characteristics of the isolate were identified as Aspergillus fumigatus by macroscopic aspects of texture, color and microscopic aspects, such as mycelium and conidium types, relationship between hyphae and fructification organs by lactophenol cotton bluemount ${ }^{3}$.

Some authorities (e.g. Infectious Diseases Society of America [IDSA]) recommend sputum Gram staining and cultures for pathogen identification ${ }^{4}$. In many clinical microbiology laboratories, the culture of respiratory tract secretions is incubated routinely for $48 \mathrm{~h}$. However, some pathogenic organisms of LRTI such as Nocardia species and fungi, need incubation for a longer period. Nocardia and fungal infections are important causes of LRTI in the immunocompromised hosts $^{5,6}$. People on chronic steroid therapy, those with cancer, organ or bone marrow transplants, or infected with human immunodeficiency virus (HIV) are at risk. The diagnosis of those infections is often based on the results of microbial pathogens analysis. As Nocardia and fungal cultures require considerable longer incubation periods, the routine respiratory secretion culture of Nocardia species and fungi is generally negative. In the two cases presented, the cultures were prolonged to 72 $h$, because Gram staining of samples demonstrated that there were pathogens present. We propose that Gram staining paired to routine culture is valuable and should be encouraged in clinical practice for the management of lower respiratory tract infections. In specific cases, especially among immunocompromised hosts, the search for atypical microbial etiologies using Gram staining, can become extraordinarily valuable

Acknowledgements: The study was supported by a program for Hundred Medical Academic Leaders of Longgang Distinction (No.200902).

Ming-yi Wang ${ }^{1^{*}}$, Xiao-tao $\mathrm{YE}^{2^{*}}$, Xiao-wen XIN ${ }^{1}$

${ }^{1}$ Department of Clinical Lab, Weihai Municipal Hospital, Dalian Medical University, Shandong, China

${ }^{2}$ Department of Clinical Lab, Longgang District Central Hospital of Shenzhen, Shenzhen, China 


\section{References}

1. Pratter MR, Irwin RS. Clinical value of the Gram-stain smear of respiratory secretions. Chest 1985; 88: 163-4.

2. Washington W, Stephen A, William J, Elmer K, Gary P, Paul S, et al. Koneman's Colour Atlas and Textbook of Diagnostic Microbiology: $6^{\text {th }}$ ed. Philadelphia Lippincott Williams and Wilkins; 2006; 859-940.

3. de Hoog GS, Guarro J, GeneÂ J, Figueras MJ. Atlas of Clinical Fungi, $2^{\text {nd }}$ ed. Utrecht NL, Centraalbureau voor Schimmelcultures. 2000.

4. Dalhoff K. Worldwide guidelines for respiratory tract infections: community-acquired pneumonia. Int J Antimicrob Agents 2001; 18 Suppl 1: S39-44.

5. Tuo MH, Tsai YH, Tseng HK, Wang WS, Liu CP, Lee CM. Clinical experiences of pulmonary and bloodstream nocardiosis in two tertiary care hospitals in northern Taiwan, 2000-2004. J Microbiol Immunol Infect 2008; 41: 130-6.

6. Castón JJ, Linares MJ, Gallego C, Rivero A, Font P, Solís F, et al. Risk factors for pulmonary Aspergillus terreus infection in patients with positive culture for filamentous fungi. Chest 2007; 131: 230-6.

Corresponding author:

Ming-yi Wang.

E-mail:mingyi_wang@hotmail.com

*These authors contributed equally to this work and should be considered as joint first author.

Conflict of interest: There are no conflicts of interests to be declared in this study. 